\title{
ANALYSIS OF UNKNOWN-LOCATION SIGNAL DETECTABILITY FOR REGULARIZED TOMOGRAPHIC IMAGE RECONSTRUCTION
}

\author{
Anastasia Yendiki \\ HMS/MGH/MIT Martinos Center \\ for Biomedical Imaging \\ 149 13th St. \\ Charlestown, MA 02129-2301, USA
}

\author{
Jeffrey A. Fessler* \\ University of Michigan \\ EECS Department \\ 1301 Beal Ave. \\ Ann Arbor, MI 48109-2122, USA
}

\begin{abstract}
Our goal is to optimize regularized image reconstruction methods for emission tomography with respect to the task of detecting small lesions of unknown location in the reconstructed images. We consider model observers whose decisions are based on finding the maximum value of a local test statistic over all possible lesion locations. We use tail probability approximations by Adler (AAP 2000) and Siegmund and Worsley (AS 1995) to evaluate the probabilities of false alarm and detection respectively for the observers of interest. We illustrate how these analytical tools can be used to optimize regularization with respect to the performance (at low probability of false alarm operating points) of a maximum channelized non-prewhitening observer.
\end{abstract}

\section{INTRODUCTION}

Tomographic reconstruction methods typically involve userspecified parameters that control the noise-resolution tradeoff in the reconstructed images, e.g., regularization parameters in penalized-likelihood reconstruction. We would like to have a fast, analytical method for choosing such parameters optimally based on some objective image quality criterion.

Several applications of emission tomography involve the detection of a spatially localized target signal in an image reconstructed from noisy data. We are interested in optimizing the parameters of regularized image reconstruction methods with respect to the detectability of such signals in the reconstructed images. In clinical practice, these detection tasks are typically performed by human observers. However, the detection performance of humans does not lend itself to analytical optimization. Thus we turn to the mathematical observers that have been proposed in the literature to model human performance $[1, \S 14.3]$. To reflect clinical tasks more realistically, it is of particular interest to consider detection tasks where the location of the target signal is not known a priori.

Location uncertainty complicates the analysis of detectability. Following convention, we focus on model observers whose

${ }^{*}$ This work was supported in part by NCI grant P01 CA87634. decisions are based on the maximum value of a linear local test statistic over all possible signal locations. The exact distribution of the maximum of a correlated random field has the form of a multiple integral, which is difficult to compute. The "brute-force" approach to evaluating the performance of these observers would be to perform time-consuming tomographic reconstructions of Monte Carlo simulated projection data and produce realizations of the maximum test statistic from the reconstructed images. To avoid this, investigators have proposed analytical approximations of the moments of the local test statistics and used them to directly produce Monte Carlo simulated realizations of the maximum test statistic $[2,3]$.

We follow an alternative approach which obviates the need for simulations. Although the exact expression for the distribution of the maximum is complicated, simple approximations of this distribution at high thresholds do exist for correlated Gaussian random fields [4]. In [5] we used these approximations to evaluate the probability of detection of the maximum observer at low probabilities of false alarm. However, that analysis assumes (local) stationarity, so it is more accurate for the signal-absent than the signal-present hypothesis. Here we adopt instead the approach of [6] for the signalpresent analysis. We illustrate how these tools can be combined to analyze the performance of the observers of interest. Ultimately, we would like to use this approach to tune regularization parameters locally at each pixel in the image.

\section{THE DETECTION TASK}

Let $f$ denote the true object being imaged (or its approximation in $\mathbb{R}^{n_{\mathrm{p}}}$ ). The object $f$ consists of a background $\boldsymbol{f}_{b}$ and it may or may not also contain a spatially localized signal of interest. We assume that, when the target signal is present in the object, it is centered at one of a finite set of locations $\ell=1, \ldots, n_{\mathrm{L}}$. We denote the signal centered at location $\ell$ by $\boldsymbol{f}_{s, \ell}$. The task at hand is to decide among $n_{\mathrm{L}}+1$ hypotheses:

$$
\begin{array}{lll}
H_{0} & : \quad \boldsymbol{f}=\boldsymbol{f}_{b} \quad \text { (signal absent) } \\
H_{\ell} & \left.: \quad \boldsymbol{f}=\boldsymbol{f}_{b}+\boldsymbol{f}_{s, \ell} \quad \text { (signal present at location } \ell\right)
\end{array}
$$


$\ell=1, \ldots, n_{\mathrm{L}}$. The background $\boldsymbol{f}_{b}$ is generally random with mean and covariance $\overline{\boldsymbol{f}}_{b}$ and $\mathcal{K}_{b}$ respectively. We assume for simplicity that the target signal is random only in its location.

The decision is based on a noisy measurement $\boldsymbol{y}$. When $\boldsymbol{y}$ is acquired by a tomographic imaging system, its unconditional mean and covariance can be written, respectively,

$$
\begin{aligned}
\mathrm{E}[\boldsymbol{y}] & =\mathcal{A E}[\boldsymbol{f}]+\boldsymbol{r} \\
\operatorname{Cov}\{\boldsymbol{y}\} & =\operatorname{diag}\{\mathcal{A} \mathrm{E}[\boldsymbol{f}]+\boldsymbol{r}\}+\mathcal{A K}_{b} \mathcal{A}^{\prime},
\end{aligned}
$$

where the linear operator $\mathcal{A}$ models the imaging system and the vector $\boldsymbol{r}$ represents scatter and/or random coincidences. Both $\mathcal{A}$ and $\boldsymbol{r}$ are assumed deterministic and known. The signal intensity is assumed weak with respect to the background intensity, so we can write $\Pi \triangleq \operatorname{diag}\{\mathcal{A E}[\boldsymbol{f}]+\boldsymbol{r}\} \approx$ $\operatorname{diag}\left\{\boldsymbol{\mathcal { A }} \overline{\boldsymbol{f}}_{b}+\boldsymbol{r}\right\}$. We focus on observer models that are applied on a reconstructed image $\hat{\boldsymbol{f}}$, obtained from $\boldsymbol{y}$ by

$$
\hat{\boldsymbol{f}}(\boldsymbol{y})=\mathcal{Z} \boldsymbol{y}
$$

for some linear reconstructor $\mathcal{Z}$. Common tomographic reconstruction techniques can be approximated as linear around the signal when the latter appears on a background that is sufficiently high to render the non-negativity constraint inactive.

Following convention, we focus on observers whose decisions rely on computing a local test statistic $t_{\ell}=t_{\ell}(\hat{\boldsymbol{f}}(\boldsymbol{y}))$ for each candidate location $\ell$ and comparing the maximum,

$$
t_{\max }=\max _{\ell=1, \ldots, n_{\mathrm{L}}} t_{\ell}
$$

to a data-independent threshold $\tau$. If $t_{\max }>\tau$, it is decided that the signal is present, otherwise it is decided that the signal is absent. Our goal is to optimize the reconstructor $\mathcal{Z}$ around each location in the image with respect to the detection performance of such an observer. We quantify the observer's performance by a ROC curve defined around a given location $\ell \in\left\{1, \ldots, n_{\mathrm{L}}\right\}$. This curve plots the probability of detection,

$$
P_{\mathrm{D}}(\tau) \triangleq \mathrm{P}\left\{t_{\max } \geq \tau \mid H_{\ell}\right\}, \text { for some } \ell \in\left\{1, \ldots, n_{\mathrm{L}}\right\},
$$

versus the probability of false alarm,

$$
P_{\mathrm{FA}}(\tau) \triangleq \mathrm{P}\left\{t_{\max } \geq \tau \mid H_{0}\right\} .
$$

The curve is traced by varying the decision threshold $\tau$.

\section{ASYMPTOTIC DISTRIBUTION OF $t_{\max }$}

To trace the ROC curve for $t_{\max }$, one must calculate the CDF of $t_{\max }$, from which threshold-exceeding probabilities such as (6) and (7) can then be obtained. Although a closed-form expression for such probabilities is generally not available when the $t_{\ell}$ 's are correlated, approximate expressions for high values of the threshold $\tau$ have been derived.

By analyzing the Euler characteristic of excursion sets, Adler has developed approximations for the distribution tails of the maximum of some correlated random fields [4]. In particular, let $t_{\max }=\max _{\boldsymbol{x} \in S} T(\boldsymbol{x})$ be the maximum value of a stationary 2-D Gaussian random field $T(\boldsymbol{x})=T\left(x_{1}, x_{2}\right)$ with zero mean, variance $\sigma_{T}^{2}$, and autocovariance function $R_{T}(\boldsymbol{x})=R_{T}\left(x_{1}, x_{2}\right)$, over a set $S$. Then the probability of $t_{\max }$ exceeding a high threshold $\tau$ is approximately

$$
\mathrm{P}\left\{t_{\max } \geq \tau\right\} \approx \sum_{d=0}^{2} R_{d}(S) \rho_{d}\left(\tau ; \sigma_{T}, \Lambda_{T}\right),
$$

where $\Lambda_{T}$ is the matrix with $i j$-th element equal to $\left\{\Lambda_{T}\right\}_{i j}=$ $-\partial^{2} R_{T}(0,0) / \partial x_{i} \partial x_{j}, i, j=1,2$. When the search area $S$ is a disk of radius $r$, we have $R_{0}(S)=1, R_{1}(S)=\pi r$, and $R_{2}(S)=\pi r^{2}$. Finally, $\rho_{0}\left(\tau ; \sigma_{T}, \Lambda_{T}\right) \triangleq 1-\Phi\left(\tau / \sigma_{T}\right)$, $\rho_{1}\left(\tau ; \sigma_{T}, \Lambda_{T}\right) \triangleq \frac{\left|\operatorname{det} \Lambda_{T}\right|^{1 / 4}}{2 \pi \sigma_{T}} e^{-\tau^{2} / 2 \sigma_{T}^{2}}$, and $\rho_{2}\left(\tau ; \sigma_{T}, \Lambda_{T}\right) \triangleq$ $\frac{\left|\operatorname{det} \Lambda_{T}\right|^{1 / 2}}{(2 \pi)^{3 / 2} \sigma_{T}^{3}} \tau e^{-\tau^{2} / 2 \sigma_{T}^{2}}$, where $\Phi(\cdot)$ is the standard normal CDF. Approximations of the form (8) have been applied to the problem of detecting activation in functional neuroimaging, yielding satisfactory accuracy for tail probabilities as high as 0.2 [7].

The analysis leading to (8) assumes a continuous random field. For fields defined solely on a lattice, the results hold asymptotically as the lattice becomes finer [4]. Thus, one can apply (8) to approximate the tail distribution of the maximum test statistic in (5), if the discrete local test statistics $t_{\ell}, \ell=1, \ldots, n_{\mathrm{L}}$ are stationary with known mean and autocovariance. Under the signal-absent hypothesis, the $t_{\ell}$ 's may be considered stationary to within the accuracy of the local shift invariance approximations discussed in section 4 . The same can be said in terms of the second-order statistics of the $t_{\ell}$ 's under the signal-present hypothesis. However, the mean of the $t_{\ell}$ 's cannot be considered constant throughout the search area in the presence of a spatially localized target signal.

An alternative approach to the signal-present analysis follows the argument of Siegmund and Worsley in [6]. This approach assumes that, in the signal-present case, $(i)$ The maximum is most likely to occur near $\boldsymbol{x}_{\ell}$, i.e., near the center of the target signal, and (ii) In the immediate neighborhood of the target signal, the field $T(\boldsymbol{x})$ can be approximated as quadratic in $\boldsymbol{x}$. These assumptions lead to the following approximation:

$$
\begin{aligned}
\mathrm{P}\left\{t_{\max }>\tau \mid H_{\ell}\right\} \approx 1-\Phi\left(\frac{\tau-\mu_{T}\left(\boldsymbol{x}_{\ell}\right)}{\sigma_{T}}\right) \\
+\phi\left(\frac{\tau-\mu_{T}\left(\boldsymbol{x}_{\ell}\right)}{\sigma_{T}}\right) \frac{1}{\sigma_{T}}\left[\frac{\partial^{2} R_{T}(\mathbf{0})}{\partial^{2} x_{i}}\right] /\left[\frac{\partial^{2} \mu_{T}\left(\boldsymbol{x}_{\ell}\right)}{\partial^{2} x_{i}}\right],(9)
\end{aligned}
$$

where $\phi(\cdot)$ the standard normal PDF and $\mu_{T}(\boldsymbol{x}) \triangleq \mathrm{E}\left[T(\boldsymbol{x}) \mid H_{\ell}\right]$.

We will combine here (8) and (9) with locally shift-invariant approximations to compute, respectively, the probabilities of false alarm and detection for a commonly used observer model.

\section{MOMENTS OF THE LOCAL TEST STATISTICS}

The observer models proposed in the literature to predict human performance use linear channelized local test statistics. 
For every candidate location $\ell$, these models apply some $l o$ cal template $\boldsymbol{w}_{\ell} \in \mathbb{R}^{M}$ to the output $\hat{\boldsymbol{c}}_{\ell}$ of $M$ bandpass filters:

$$
t_{\ell}=\boldsymbol{w}_{\ell}^{\prime} \hat{\boldsymbol{c}}_{\ell}, \quad \hat{\boldsymbol{c}}_{\ell}=\mathcal{C}_{\ell}^{\prime}\left(\hat{\boldsymbol{f}}-\mathrm{E}\left[\hat{\boldsymbol{f}}_{b}\right]\right)+\boldsymbol{\varepsilon}_{\ell},
$$

where $\mathcal{C}_{\ell}=\left[\mathcal{C}_{1, \ell}, \ldots, \mathcal{C}_{M, \ell}\right]$ consists of $M$ operators, each of which applies a bandpass filter and samples the output at location $\ell$. The internal noise vector $\varepsilon_{\ell} \in \mathbb{R}^{M}$ models inherent uncertainty in the observer's decisions and is usually modeled as zero-mean and Gaussian with some covariance matrix $\boldsymbol{\Pi}_{\varepsilon_{\ell}}$. As in [2,3], the mean of the reconstructed background $\hat{\boldsymbol{f}}_{b} \triangleq \mathcal{Z}\left(\mathcal{A} \boldsymbol{f}_{b}+\boldsymbol{r}\right)$ is subtracted in (10) because the observer chooses the most suspicious location by comparing intensities relative to the background, rather than absolute intensities.

Let $t \in \mathbb{R}^{n_{\mathrm{L}}}$ be the vector containing the local test statistics $t_{\ell}, \ell=1, \ldots, n_{\mathrm{L}}$, and let $\mathcal{V}=\left[\boldsymbol{v}_{1}, \ldots, \boldsymbol{v}_{n_{\mathrm{L}}}\right]$ contain the image-domain local templates $\boldsymbol{v}_{\ell}=\mathcal{C}_{\ell} \boldsymbol{w}_{\ell}$. Combining (2) and (3) with the linear reconstructor (4) yields the mean of $\boldsymbol{t}$ under the signal absent and present hypotheses respectively:

$$
\boldsymbol{\mu}_{t \mid H_{0}}=\mathbf{0}, \quad \boldsymbol{\mu}_{t \mid H_{\ell}}=\mathcal{V}^{\prime} \mathcal{Z} \mathcal{A} \boldsymbol{f}_{s, \ell}, \quad \ell=1, \ldots, n_{\mathrm{L}},
$$

and the covariance of $\boldsymbol{t}$ under any of the $n_{\mathrm{L}}+1$ hypotheses:

$$
\boldsymbol{\Pi}_{t}=\mathcal{V}^{\prime} \mathcal{Z} \boldsymbol{\Pi}_{y} \mathcal{Z}^{\prime} \mathcal{V}+\operatorname{diag}\left\{\boldsymbol{w}_{\ell}^{\prime} \boldsymbol{\Pi}_{\boldsymbol{\varepsilon}_{\ell}} \boldsymbol{w}_{\ell}, \ell=1, \ldots, n_{\mathrm{L}}\right\}
$$

with $\Pi_{y}=\Pi+\mathcal{A} \mathcal{K}_{b} \mathcal{A}^{\prime}$. Regardless of the exact distribution of the data, the local test statistics can be approximated as Gaussian due to their linearity and the central limit theorem. Thus we will use (8) and (9) to evaluate the probabilities of false alarm and detection respectively. Both approximations assume stationarity in terms of the second-order moments of the $t_{\ell}$ 's. For a typical shift-variant tomographic system, the $t_{\ell}$ 's are not globally stationary. However, we will consider the system to be locally shift-invariant and the object background to be locally stationary over a small area around each candidate location $\ell$. Then the second-order moments of the $t_{\ell}$ 's can be approximated as constant in that local region.

We illustrate the use of this approach for a reconstructor $\mathcal{Z}$ of the unconstrained quadratically penalized weighted least-squares (QPWLS) family with uniform regularization:

$$
\mathcal{Z}=(\mathcal{F}+\beta \mathcal{R})^{-1} \mathcal{A}^{\prime} \Pi^{-1},
$$

where $\mathcal{F} \triangleq \mathcal{A}^{\prime} \Pi^{-1} \mathcal{A}$ the Fisher information operator, $\mathcal{R}$ the regularization operator and $\beta$ the user-specified regularization parameter that controls resolution in the reconstructed image. Our goal is to choose $\beta$ for optimal detection performance.

We assume that the target signal $\boldsymbol{f}_{s, \ell}$ takes the same shape at any candidate location $\ell$. That is, $\boldsymbol{f}_{s, \ell}$ is a copy of a common signal profile $\boldsymbol{f}_{s}$ shifted to location $\ell$. Similarly, we assume that the responses of the observer's channels at different locations $\ell$ are shifted copies of each other. Let $\boldsymbol{X}_{0} \in \mathbb{C}^{n_{\mathrm{p}}}$ be the spectrum of $\boldsymbol{f}_{s}$, when the latter is assumed centered at $(0,0)$, and let $\boldsymbol{T}_{0} \in \mathbb{C}^{n_{\mathrm{p}} \times M}$ contain in its $M$ columns the frequency responses of the observer's $M$ channels, when their impulse responses are assumed centered at $(0,0)$.
We now assume that the target signal profile $f_{s}$ is welllocalized in space. Similarly to [2,3], we approximate the system as locally shift-invariant and the object as locally stationary under all hypotheses around any specific location $\ell$. Then, for the QPWLS reconstructor (13), we can approximate within some small spatial extent around location $\ell$ :

$$
\mathcal{Z} \mathcal{A} \approx \mathcal{U}^{-1} \boldsymbol{H} \mathcal{U}, \quad \mathcal{Z} \Pi_{y} \mathcal{Z}^{\prime} \approx \mathcal{U}^{-1} G \mathcal{U}
$$

where $\boldsymbol{H} \triangleq \operatorname{diag}\left\{\lambda_{k} /\left(\lambda_{k}+\beta \omega_{k}\right), k=1, \ldots, n_{\mathrm{p}}\right\}, \quad \boldsymbol{G} \triangleq$ $\operatorname{diag}\left\{\left(\lambda_{k}+\lambda_{k}^{2} \nu_{k}\right) /\left(\lambda_{k}+\beta \omega_{k}\right)^{2}, k=1, \ldots, n_{\mathrm{p}}\right\}$ and where the $\lambda_{k}$ 's, $\nu_{k}$ 's, and $\omega_{k}$ 's contain the local frequency responses of $\mathcal{F}, \mathcal{K}_{b}$, and $\mathcal{R}$ respectively at the location $\ell$.

The maximum channelized non-prewhitening (MaCNPW) observer has been shown to predict human observer performance in some unknown-location tasks [8]. The local template applied by this observer at each location $\ell$ is

$$
\boldsymbol{w}_{\mathrm{CNPW}, \ell} \triangleq \mathrm{E}\left[\hat{\boldsymbol{c}}_{\ell} \mid H_{1, \ell}\right]-\mathrm{E}\left[\hat{\boldsymbol{c}}_{\ell} \mid H_{0}\right]=\mathcal{C}_{\ell}^{\prime} \mathcal{Z} \mathcal{A} \boldsymbol{f}_{s, \ell}
$$

For a reconstructor $\mathcal{Z}$ of the QPWLS family (13), applying the locally-shift invariant approximation of (14) yields the following approximation to the MaCNPW local template in (15):

$$
\boldsymbol{w}_{\mathrm{CNPW}, \ell} \approx \boldsymbol{T}_{0}^{\prime} \boldsymbol{H} \boldsymbol{X}_{0}
$$

Let the $\lambda_{k}$ 's, $\omega_{k}$ 's, and $\nu_{k}$ 's contain the local frequency responses of the respective operators at location $\ell_{c}$, corresponding to the center of the observer's search area. We assume that these responses capture the approximate behavior of the operators throughout the search area, if the latter is not too big. This implies that the MaCNPW templates in (16) are approximately shift-invariant throughout the search area, i.e., $\boldsymbol{w}_{\ell} \approx$ $\boldsymbol{w}_{\ell_{c}}, \ell=1, \ldots, n_{\mathrm{L}}$. Substituting the frequency-domain representations (14) into the moments (11)-(12) yields

$$
\begin{aligned}
\boldsymbol{\mu}_{t \mid H_{\ell}} & \approx n_{\mathrm{p}} \mathcal{I}_{\mathrm{SA}}^{\prime} \mathcal{U}^{-1} \boldsymbol{\Phi} \mathcal{U} \boldsymbol{e}_{\ell}, \quad \ell=1, \ldots, n_{\mathrm{L}} \\
\boldsymbol{\Pi}_{t} & \approx n_{\mathrm{p}} \mathcal{I}_{\mathrm{SA}}^{\prime} \mathcal{U}^{-1} \boldsymbol{\Psi} \mathcal{U} \mathcal{I}_{\mathrm{SA}}+\left(\boldsymbol{w}_{\ell_{c}}^{\prime} \boldsymbol{\Pi}_{\boldsymbol{\varepsilon}_{\ell_{c}}} \boldsymbol{w}_{\ell_{c}}\right) \mathcal{I}(18)
\end{aligned}
$$

where $\mathcal{I}_{\mathrm{SA}} \triangleq\left[\boldsymbol{e}_{1}|\ldots| \boldsymbol{e}_{n_{\mathrm{L}}}\right]$ has as its columns the impulses $\boldsymbol{e}_{\ell}, \ell=1, \ldots, n_{\mathrm{L}}$, corresponding to the $n_{\mathrm{L}}$ locations in the search area, $\boldsymbol{\Phi} \triangleq \operatorname{diag}\left\{V_{k}^{*} X_{k} \lambda_{k} /\left(\lambda_{k}+\beta \omega_{k}\right), k=1, \ldots, n_{\mathrm{p}}\right\}$, $\Psi \triangleq \operatorname{diag}\left\{\left|V_{k}\right|^{2} \lambda_{k}\left(1+\lambda_{k} \nu_{k}^{\ell}\right) /\left(\lambda_{k}+\beta \omega_{k}\right)^{2}, k=1, \ldots, n_{\mathrm{p}}\right\}$, and where $V_{k}, X_{k}$ are the $k$ th elements of $\boldsymbol{V}_{0} \triangleq \boldsymbol{T}_{0} \boldsymbol{w}_{\ell_{c}}$ and $\boldsymbol{X}_{0}$ respectively.

\section{PRELIMINARY RESULTS AND DISCUSSION}

We obtain $\sigma_{T}, \Lambda_{T}$ from the local Fourier approximation (18) and use them in the approximation (8) to calculate the probability of false alarm (7) at high detection thresholds $\tau$. We also obtain $\mu_{T}\left(\boldsymbol{x}_{\ell_{c}}\right)$ from the local Fourier approximation of the mean (17) and use it in the approximation (9) to calculate the probability of detection (6). We obtain the two derivatives required in (9) by applying finite differences to (18) and (17). 


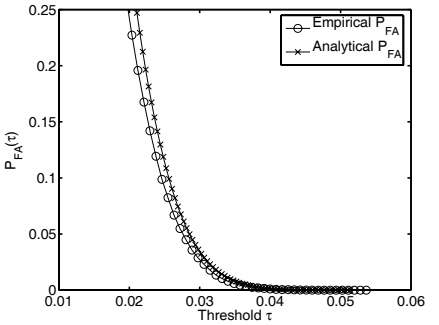

(a) $P_{\mathrm{FA}}$ (Search diam. 7 pixels)

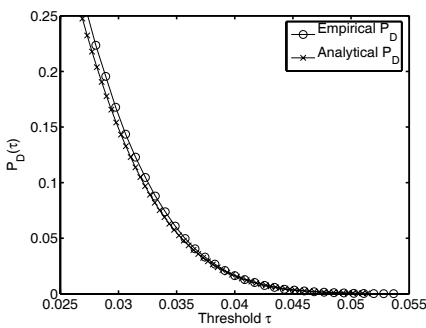

(c) $P_{\mathrm{D}}$ (Search diam. 7 pixels)

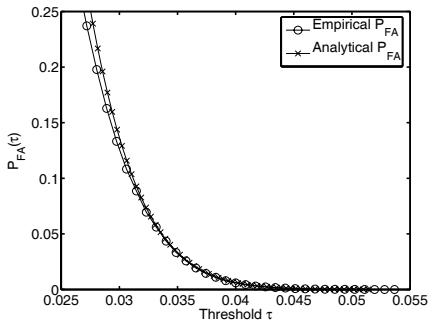

(b) $P_{\mathrm{FA}}$ (Search diam. 15 pixels)

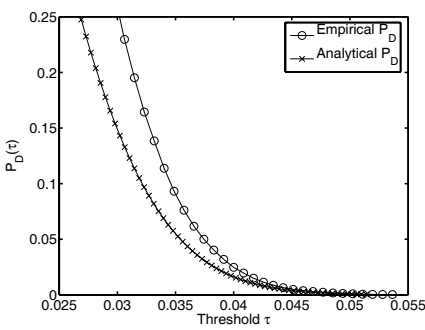

(d) $P_{\mathrm{D}}$ (Search diam. 15 pixels)
Fig. 1. Empirical and analytical probabilities of false alarm $P_{\mathrm{FA}}(\tau)$ and detection $P_{\mathrm{D}}(\tau)$ versus detection threshold $\tau$.

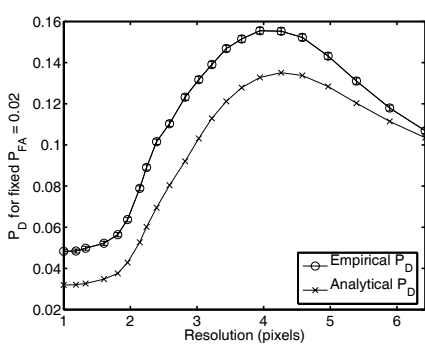

(a) Search diameter: 7 pixels

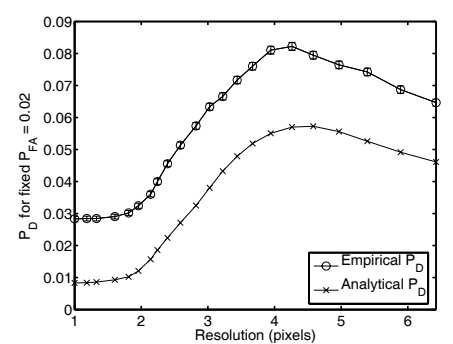

(b) Search diameter: 15 pixels
Fig. 2. Plot of $P_{\mathrm{D}}$ (with error bars) versus QPWLS resolution for a fixed $P_{\mathrm{FA}}=0.02$.

As an example, we consider a 2-D PET system model with image size $128 \times 128$ and an anthropomorphic chest phantom as the mean background $\bar{f}_{b}$. The background $\boldsymbol{f}_{b}$ has a Gaussian autocorrelation function. The target signal $\boldsymbol{f}_{s, \ell}$ has a known Gaussian profile but unknown location $\ell$. We consider a MaCNPW observer with square bandpass channels, no internal noise, and a disk-shaped search area. We calculate $P_{\mathrm{D}}(\tau)$ and $P_{\mathrm{FA}}(\tau)$ analytically for different values of the threshold $\tau$. We repeat this for various values of $\beta$. Fig. 1 compares these results to the empirical probabilities obtained from simulations, for an intermediate QPWLS resolution of 3 pixels and for two different search area sizes. We interpolate these results to find the probability of detection $P_{\mathrm{D}}$ at a fixed value of the probability of false alarm $P_{\mathrm{FA}}=0.02$ for every $\beta$. Fig. 2 shows the linearly interpolated $P_{\mathrm{D}}$ vs. QPWLS resolution. As seen in Fig. 1(c)-1(d), the quadratic approximation implied in (9) is more accurate for a search diameter of 7 pixels, which is comparable to the support of the target signal, than for a larger search area. Even if the analytical approximations are not always accurate at predicting the value of the probability of detection, they are maximized at nearly the same QPWLS resolution as the empirical curves. Thus, analytically computed plots like the ones in Fig. 2 can be used for tuning the regularization parameter $\beta$ (i.e., the resolution) of the QPWLS reconstructor to optimize detection performance locally, much faster than with simulation-based methods. For further discussion of these results, we refer the reader to [9].

\section{REFERENCES}

[1] H. H. Barrett and K. J. Myers, Foundations of image science, Wiley, New York, 2003.

[2] P. K. Khurd and G. R. Gindi, "LROC model observers for emission tomographic reconstruction," in Proc. SPIE 5372, Medical Imaging 2004: Image Perception, $O b-$ server Performance, and Technology Assessment, 2004, pp. 509-20.

[3] J. Qi and R. H. Huesman, "Fast approach to evaluate MAP reconstruction for lesion detection and localization," in Proc. SPIE 5372, Medical Imaging 2004: Image Perception, Observer Performance, and Technology Assessment, 2004, pp. 273-82.

[4] R. J. Adler, "On excursion sets, tube formulas and maxima of random fields," Ann. Appl. Probab., vol. 10, no. 1, pp. 1-74, Feb. 2000.

[5] A. Yendiki and J. A. Fessler, "Analysis of observer performance in detecting signals with location uncertainty for regularized tomographic image reconstruction," in Proc. IEEE Nuc. Sci. Symp. Med. Im. Conf., 2004, vol. 4, pp. 2620-4.

[6] D. O. Siegmund and K. J. Worsley, “Testing for a signal with unknown location and scale in a stationary Gaussian random field," Ann. Stat., vol. 23, no. 2, pp. 608-39, Apr. 1995.

[7] K. J. Worsley, S. Marrett, P. Neelin, A. C. Vandal, K. J. Friston, and A. C. Evans, "A unified statistical approach for determining significant signals in images of cerebral activation," Hum. Brain Map., vol. 4, no. 1, pp. 58-73, 1996.

[8] H. C. Gifford, P. H. Pretorius, and M. A. King, "Comparison of human- and model-observer LROC studies," in Proc. SPIE 5034: Medical Imaging 2003: Image Perc., Obs. Perf. and Tech. Assess., 2003, pp. 112-22.

[9] A. Yendiki, Analysis of signal detectability in statistically reconstructed tomorgaphic images, Ph.D. thesis, Univ. of Michigan, Ann Arbor, MI 48109-2122, 2005. 